\title{
BOUNDEDNESS THEOREM FOR A NONLINEAR MATHIEU EQUATION*
}

\author{
By JOSEPH GENIN (Purdue University) \\ AND \\ JOHN S. MAYBEE (University of Colorado)
}

1. Introduction. In his book on dynamic stability Bolotin [1] presents, after considerable development, the nonlinear differential equation describing the motion of a parametrically excited column

$$
f^{\prime \prime}+2 \epsilon f^{\prime}+\Omega^{2}(1-2 \mu \cos \theta t) f+\psi\left(f, f^{\prime}, f^{\prime \prime}\right)=0,
$$

where a prime represents differentiation with respect to time, $\epsilon$ is the coefficient of the linear damping force, $\Omega$ is the frequency parameter, $\mu$ is the excitation parameter and $\theta$ is the frequency of the forcing function. More specifically, $\Omega$ is a parameter representing the system's natural frequency and $\mu$ is a parameter related to the magnitude of the forcing function. The function $\psi$ can be shown to take the form

$$
\psi\left(f, f^{\prime}, f^{\prime \prime}\right)=\gamma f^{3}+2 \epsilon_{L} f^{2} f^{\prime}+2 \kappa\left[f f^{\prime \prime}+\left(f^{\prime}\right)^{2}\right] f,
$$

where $\gamma$ is the coefficient of the nonlinear elastic restoring force, $\epsilon_{L}$ is the coefficient of the nonlinear damping force, and $\kappa$ is the coefficient of the nonlinear inertia force. The nonnegative constants $\epsilon, \gamma, \epsilon_{L}$ and $\kappa$ characterize internal and hence natural properties of the column. However, it should be noted that one can formulate equivalent external mechanisms to produce the same linear and nonlinear effects.

An inspection of (1) reveals that it is a nonlinear form of the well-known Mathieu equation. That is, if the coefficient of linear damping $\epsilon$ and the nonlinear function $\psi$ vanish then the equation reduces to a Mathieu equation (assuming that the frequency and the excitation parameters are constants). The analysis of the Mathieu equation is, of course, well documented, as is the influence of the linear damping term on the regions of dynamic stability of the Mathieu equation (see Bolotin [1], McLachlan [2], for example.) Thus the present interest in Eq. (1) focuses about the effect of the nonlinear function $\psi$ on the boundedness and stability of the system.

In dealing with the system of Eqs. (1), (2) Bolotin uses the method of slowly varying amplitudes, and in so doing reduces the system to one which may be regarded as qausilinear. With this procedure he is able to extract useful information concerning the relationship between the amplitude of vibration of the system and the parameters $\theta, \Omega$ and $\mu$. However, he avoided the important question of the boundedness of solutions for the system.

The purpose of the present note is to fill this gap with the following theorem on boundedness of solutions.

\footnotetext{
* Received September 15, 1969; revised version received November 7, 1969.
} 
Theorem. Let $f$ be any solution of the initial value problem for the differential equation (1), (2) in which all constants are nonnegative, and if $\kappa \neq 0$ the inequality

$$
\kappa \leq \epsilon_{L} / 2 \epsilon
$$

is satisfied. Then there exist positive constants $\zeta_{0}$ and $\eta_{0}$ such that

$$
|f| \leq \zeta_{0}, \quad\left|f^{\prime}\right| \leq \eta_{0}
$$

for all nonnegative $t$.

Our proof of this theorem is achieved through the use of an energy identity. We have discussed similar identities in [3], [4] and [5].

2. Energy identity. Let us consider the initial value problem for the system (1), (2) and establish the basic energy identity we wish to use. We begin by placing (2) into (1) and multiplying the resulting equation by $f^{\prime}$ to obtain

$$
\frac{1}{2}\left(f^{\prime 2}\right)^{\prime}+2 \epsilon f^{\prime 2}+\Omega^{2}(1-2 \mu \cos \theta t)\left(f^{2}\right)^{\prime} / 2+(\gamma / 4)\left(f^{4}\right)^{\prime}+2 \epsilon_{L} f^{2} f^{\prime 2}+\kappa\left(\left(f f^{\prime}\right)^{2}\right)^{\prime}=0 .
$$

Now, multiplying by $\epsilon$ leads to

$$
\begin{aligned}
\epsilon\left(f f^{\prime}\right)^{\prime}-\epsilon f^{\prime 2}+\epsilon^{2}\left(f^{2}\right)^{\prime}+\epsilon \Omega^{2}(1-2 \mu \cos \theta t) f^{2} & \\
& +\epsilon \gamma f^{4}+\left(\epsilon \epsilon_{L} / 2\right)\left(f^{4}\right)^{\prime}+2 \kappa \epsilon\left(f^{3} f^{\prime}\right)^{\prime}-4 \kappa \epsilon f^{2} f^{\prime 2}=0 .
\end{aligned}
$$

Adding the two equations and collecting terms yields

$$
\begin{aligned}
& \left\{\frac{1}{2} f^{2}+\epsilon f f^{\prime}+\left(\Omega^{2} / 2\right) f^{2}+(\gamma / 4) f^{4}+\kappa f^{2} f^{\prime 2}+\epsilon^{2} f^{2}+\left(\epsilon \epsilon_{L} / 2\right) f^{4}+2 \kappa \epsilon f^{3} f^{\prime}\right\}^{\prime} \\
& \quad+\epsilon f^{\prime 2}-\Omega^{2} \mu\left(f^{2}\right)^{\prime} \cos \theta t+2 \epsilon_{L} f^{2} f^{\prime 2} \\
& \quad+\epsilon \Omega^{2}(1-2 \mu \cos \theta t) f^{2}+\epsilon \gamma f^{4}-4 \epsilon \kappa f^{2} f^{\prime 2}=0 .
\end{aligned}
$$

From both the mathematical and physical point of view the case $\mu>\frac{1}{2}$ is the most significant, for it represents the case when the magnitude of the harmonic forcing function has a large influence on the solution. That is, in the development of Eq. (1) the forcing function on the column was postulated in the form

$$
P=P_{0}+P_{1} \cos \tau t
$$

from which evolved the constant

$$
\mu=P_{1} /\left(2\left(P^{*}-P_{0}\right)\right), \quad\left(\mu>0 ; P_{0} \neq P^{*}\right)
$$

where $P^{*}$ is the linear static Euler critical buckling load. Thus it is seen that as $\mu \rightarrow 0$ we approach a static loading of the column.

From the foregoing it is seen that the conditions for studying the boundedness of the parametrically excited system (1), (2) are that all of the constants be nonnegative.

Returning now to the last energy equation above, we note the identity

$$
\left(-\Omega^{2} \mu f^{2} \cos \theta t\right)^{\prime}=-\mu \Omega^{2}\left(f^{2}\right)^{\prime} \cos \theta t+\mu \Omega^{2} \theta f^{2} \sin \theta t .
$$

To simplify the handling of the equations let us set

$$
\begin{aligned}
E(f, t)=f^{\prime 2} / 2+\epsilon f f^{\prime}+\left[\left(\Omega^{2} / 2\right)(1-2 \mu \cos \theta t)+\right. & \left.\epsilon^{2}\right] f^{2} \\
& +\left(\gamma / 4+\epsilon \epsilon_{L} / 2\right) f^{4}+\kappa f^{2} f^{2}+2 \kappa \epsilon f^{3} f^{\prime}
\end{aligned}
$$

and

$$
F(f, t)=\epsilon f^{\prime 2}+\Omega^{2}[\epsilon(1-2 \mu \cos \theta t)-\mu \theta \sin \theta t] f^{2}+2\left(\epsilon_{L}-2 \epsilon \kappa\right) f^{2} f^{\prime 2}+\epsilon \gamma f^{4} .
$$


Thus we have, after integration,

$$
E\left(f, t_{2}\right)+\int_{t_{2}}^{t_{\bullet}} F(f, s) d s=E\left(f, t_{1}\right)
$$

for any $0 \leq t_{1}<t_{2}$.

3. Inertial and damping constants. In order to work with the energy identity (3) we shall assume that the term

$$
2\left(\epsilon_{L}-2 \epsilon \kappa\right) f^{2} f^{\prime 2}
$$

in the functional $F(f, t)$ is positive. Hence when $\kappa>0$ we assume that

$$
\epsilon_{L} /(2 \epsilon) \geq \kappa .
$$

For the case when $\kappa=0$ there are no restrictions.

The requirement (4) can be further justified as follows. Write

where

$$
E(f, t)=E_{1}(f, t)+E_{2}(f, t)
$$

$$
E_{2}(f, t)=\left(\epsilon \epsilon_{L} / 2\right) f^{4}+\kappa f^{2} f^{\prime 2}+2 \epsilon \kappa f^{2}\left(f f^{\prime}\right) .
$$

We note that $E_{2}(f, t)$ is a quadratic form in $f^{2}$ and $f f^{\prime}$ and that it is nonnegative definite if and only if

$$
\left(\epsilon \epsilon_{L} / 2\right) \kappa-\epsilon^{2} \kappa^{2} \geq 0 .
$$

Hence if (4) is satisfied $E_{2}(f, t) \geq 0$. If $\kappa=0$ there are again no restrictions.

4. Boundedness analysis. Consider first the case when $F(f, t) \leq 0$. In this case we have

$$
\epsilon e f^{\prime 2}+\epsilon \gamma f^{4} \leq-\Omega^{2}[\epsilon(1-2 \mu \cos \theta t)-\mu \sin \theta t] f^{2} .
$$

Hence

$$
f^{2} \leq \frac{\Omega^{2}[\mu+\epsilon(2 \mu+1)]}{\epsilon \gamma}, \quad f^{\prime 2} \leq \frac{\Omega^{2}[\mu+\epsilon(2 \mu+1)]}{\epsilon} \leq \frac{\Omega^{4}[\mu+\epsilon(2 \mu+1)]^{2}}{\epsilon^{2} \gamma} .
$$

To pursue the other case in which $F(f, t) \geq 0$, let

$$
T_{1}=\{t \geq 0 \mid F(f, t) \leq 0\}, \quad T_{2}=\{t \geq 0 \mid F(f, t) \geq 0\} .
$$

The sets $T_{1}$ and $T_{2}$ are closed sets consisting of disjoint intervals each of which either has finite length or is of the form $\left[t_{0}, \infty\right), t_{0} \geq 0$. On the set $T_{1}$ the inequalities (6) are satisfied. The sets $T_{1}$ and $T_{2}$ have as their intersection the points (if any exist) at which $F(f, t)=0$. At these points equations (6) hold. Hence, at the left-hand endpoint of any interval of the set $T_{2}$ the function $E(f, t)$ is bounded by the maximum of the bound obtained by applying $(6)$ to estimate $E$ and the number $E(f, 0)$. Call this maximum $\zeta$.

Let $t_{0}$ be the left-hand endpoint of a typical interval of the set $T_{2}$. If $t>t_{0}$ is any point of the interval in question we deduce from (3) that

$$
E(f, t) \leq E\left(f, t_{0}\right) \leq \zeta
$$

Now we have

$$
E(f, t) \geq E_{1}(f, t)=\frac{1}{2} f^{\prime 2}+\epsilon f f^{\prime}+\left[\left(\Omega^{2} / 2\right)(1-2 \mu \cos \theta t)+\epsilon^{2}\right] f^{2}+(\gamma / 4) f^{4} .
$$


But

$$
\epsilon f f^{\prime} \geq-\frac{1}{2}\left(\sigma f^{\prime 2}+\frac{\epsilon^{2} f^{2}}{\sigma}\right) \text { for arbitrary } \sigma>0 \text {. }
$$

Thus,

$E(f, t) \geq \frac{1}{2}(1-\sigma) f^{\prime 2}+\left[\left(\Omega^{2} / 2\right)(1-2 \mu \cos \theta t)+\epsilon^{2}(1-(1 / 2 \sigma))\right] f^{2}+(\gamma / 4) f^{4}$

Setting $\sigma=1$, (8) becomes

$$
E(f, t) \geq f^{2}\left[(\gamma / 4) f^{2}+\left(\Omega^{2} / 2\right)(1-2 \mu \cos \theta t)+\epsilon^{2} / 2\right] .
$$

Thus, if

$$
(\gamma / 4) f^{2}+\left(\Omega^{2} / 2\right)(1-2 \mu \cos \theta t)+\epsilon^{2} / 2 \leq \frac{1}{2}
$$

we have

$$
f^{2} \leq(4 / \gamma)\left[\frac{1}{2}+\left(\Omega^{2} / 2\right)(2 \mu-1)-\epsilon^{2} / 2\right] .
$$

Otherwise, we have

$$
E(f, t) \geq \frac{1}{2} f^{2}
$$

and we deduce from (7) that

$$
f^{2} \leq 2 \zeta
$$

Let $\zeta^{\prime}=\max \left\{2 \zeta,(4 / \gamma)\left[\frac{1}{2}+\left(\Omega^{2} / 2\right)(\mu-1)-\epsilon^{2} / 2\right]\right\}$. Now set $\sigma=\frac{1}{2}$ in (8), from which

$$
E(f, t) \geq \frac{1}{4} f^{\prime 2}+\left[\left(\Omega^{2} / 2\right)(1-2 \mu \cos \theta t)\right] f^{2}+(\gamma / 4) f^{4}
$$

and from (7) we obtain

$$
f^{\prime 2} \leq 4 \zeta^{\prime}\left\{\left[1+\Omega^{2}(2 \mu-1)\right]+\gamma \zeta^{\prime}\right\}
$$

The bounds (9), (10) and (11) imply that $f^{2}$ and $f^{\prime 2}$ are also bounded on the set $T_{2}$. Since $T_{1} \cup T_{2}=[0, \infty)$, we have established the boundedness of $f$ and $f^{\prime}$ for all nonnegative $t$.

\section{REFERENCES}

[1] V. V. Bolotin, The dynamic stability of elastic systems, GITTL, Moscow, 1956; English transl., Holden-Day, San Francisco, Calif., 1965

[2] N. W. McLachlan, Theory and application of Mathieu functions, Clarendon Press, Oxford, 1947

[3] J. Genin and J. S. Maybee, Stability in the three dimensional whirling problem, International Journal of Nonlinear Mechanics 4, 205-215 (1969)

[4] J. Genin and J. S. Maybee, External and material damped three dimensional rotor system, International Journal of Nonlinear Mechanics, 1970 (forthcoming)

[5] J. Genin and J. S. Maybee, Introduction to applied mathematics, Holt, Rinehart and Winston, New York, 1970 